C) S. Barbary, 2015.

УДК 617.577-001-089.8

DOI 10.18019/1028-4427-2015-2-83-89

Тракционное повреждение пальца кольцом

\author{
S. Barbary \\ Университет Анри Поанкаре, Нанси, Франция
}

\title{
Finger avulsion injury with a ring
}

\begin{tabular}{c} 
S. Barbary \\
Henry Poincaré, Nancy, France \\
\hline
\end{tabular}

Приведены варианты тракционного повреждения безымянного пальца перстнем. Представлены существующие классификации. Проанализированы методики лечения, показания к ним, хирургическая техника, эффективность. Описана технология операций на костносухожильном аппарате, варианты восстановления кровообращения и иннервации отчлененного пальца.

Ключевые слова: пальцы, авульсионные повреждения, отчленение, классификация, хирургическое лечение, показания, оперативная техника, сосудистый анатомоз, реиннервация.

The variants of ring-finger avulsion injury with a ring presented. The existing classifications demonstrated. The methods of treatment, indications for them, surgical technique and efficiency analyzed. The technique of surgeries of the osteotendinous apparatus described, as well as the variants of circulation recovery and the avulsed finger innervation.

Keywords: fingers, avulsion (injuries), classification, surgical treatment, indications, operative technique, vascular anastomosis, reinnervation.

\section{ВВЕДЕНИЕ}

Речь идет о классическом отчленении безымянного пальца перстнем [1] (рис. 1). Этот вид травматизма может касаться всех пальцев, включая первый. При полном отчленении обычно происходит отрыв кожного футляра и дистальной фаланги вместе с сухожилием глубокого сгибателя пальца. После обзора классификаций, имеющихся в литературе, мы остановимся на той, которую мы применяем в текущей практике. Затем представим показания к выполнению хирургических вмешательств и оперативную методику, обращая внимание на сосуды при выполнении анастомозов. Наконец, рассмотрим опубликованные в литературе результаты, отражающие, в частности, процент выживаемости пальцев, степень восстановления двигательной активности и чувствительности пальца отдаленном периоде.
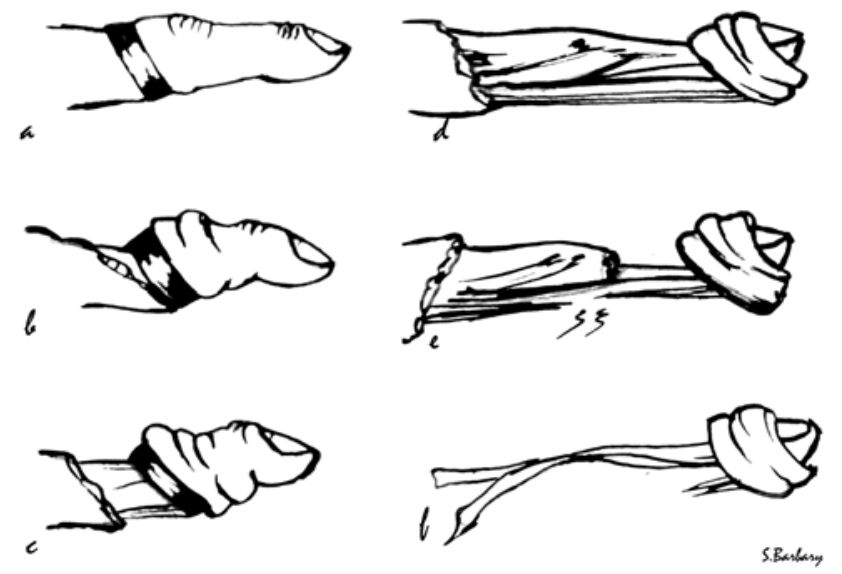

Рис. 1
Мы применяем классификацию Michon-Merle, предложенную в 1979 году, но не получившую широкого распространения в англо-саксонской литературе. Однако она легка в применении и имеет преимущество, заключающееся в дифференцировании уровней ампутации в зависимости от их сложности и функционального прогноза. Степень 1 - простое повреждение кожных покровов; 2 степень - частичное нарушение кровоснабжения без ишемии (венозной или артериальной); 3 степень - полное нарушение кровоснабжения с ишемией пальцев; 4 степень - полная ампутация пальца вследствие травматического отрыва: степень 4a - проксимальнее места прикрепления сухожилия поверхностного сгибателя пальца; степень $4 \mathrm{~b}$ - на уровне шейки 2-й фаланги или проксимального межфалангового сустава (встречается чаще других); степень 4c - полное скальпирование с отрывом мягкотканного футляра без повреждения костей. Urbaniak в 1981 году предложил классификацию, включающую 3 стадии, на эту классификацию чаще всего ссылаются в зарубежной литературе. 1 степень - повреждение тканей без нарушения кровоснабжения; 2 степень - повреждение тканей, сопровождающееся ишемией (артериальной или венозной); 3 степень - полный отрыв пальцев. В 1989 году Кау, проанализировав большое количество пациентов, вернулся к данной классификации и модифицировал ее. Он установил, что в некоторых случаях при ампутации может быть получен хороший функциональный результат, а именно, если в проксимальном межфаланговом суставе сохраняется моторика, вопрос об ампутации ставится под сомнение. В 1996 году Adani продолжил работу Кау, им была предложена классификация, включающая четыре стадии. Данная классификация имеет много общего с представленной пятнадцатью годами ранее классификацией Michon (табл. 1). 
Классификации

\begin{tabular}{|l|l|}
\hline \multicolumn{2}{|c|}{ Классификация Michon-Merle-Foucher (1979) } \\
\hline 1 степень & простое повреждение кожных покровов \\
\hline 2 степень & частичное повреждение сосудов \\
\hline 3 степень & полное нарушение кровоснабжения \\
\hline 4 степень & полное отчленение пальца: \\
\hline степень 4a & через проксимальный межфаланговый сустав с отрывом поверхностного сгибателя пальцев \\
\hline степень 4b & через шейку 2-й фаланги или дистальный межфаланговый сустав \\
\hline степень 4c & отрыв мягкотканного футляра без повреждения костей \\
\hline \multicolumn{2}{|l|}{ Классификация Urbaniak (1981) } \\
\hline 1 степень & без нарушения кровоснабжения (требуется лечение мягких тканей и скелета) \\
\hline 2 степень & полное нарушение кровоснабжения \\
\hline 3 степень & полное скальпирование \\
\hline \multicolumn{2}{|l}{ Классификация Кау, модифицированная Аdani } \\
\hline 1 степень & повреждение кожи без сосудистых нарушений \\
\hline 2 степень & нарушение кровоснабжения без повреждения кости: \\
\hline степень 2a & артериальная ишемия \\
\hline степень 2b & венозная ишемия \\
\hline 3 степень & нарушение кровоснабжения с повреждением кости: \\
\hline степень 3a & артериальная ишемия \\
\hline степень 3b & венозная ишемия \\
\hline 4 степень & полное скальпирование: \\
\hline степень 4a & проксимальнее места прикрепления поверхностного сгибателя пальца \\
\hline степень 4b & дистальнее места прикрепления поверхностного сгибателя пальца \\
\hline
\end{tabular}

\section{1. Показания}

При планировании реплантации у взрослых необходимо проинформировать пациента о сложности, длительности лечения и реабилитации, возможных осложнениях, исходах лечения (госпитализация со строгим постельным режимом в течение 5-6 дней, регулярные перевязки, возможность повторной операции, отказ от курения, период нетрудоспособности минимум до 3 месяцев и интенсивная ежедневная разработка, в отдаленном периоде возможна непереносимость охлаж- дения, гипочувствительность, ригидность суставов пальцев и т. д).

Однако при всех степенях, кроме степени 4a, выполнение реплантации возможно. Естественно, функциональный прогноз остается неясным при тракционных отчленениях выше уровня прикрепления поверхностного сгибателя пальцев. В этом случае хорошей альтернативой является незамедлительное удаление поврежденного луча с формированием четырехпалой кисти (рис. 2) [5].
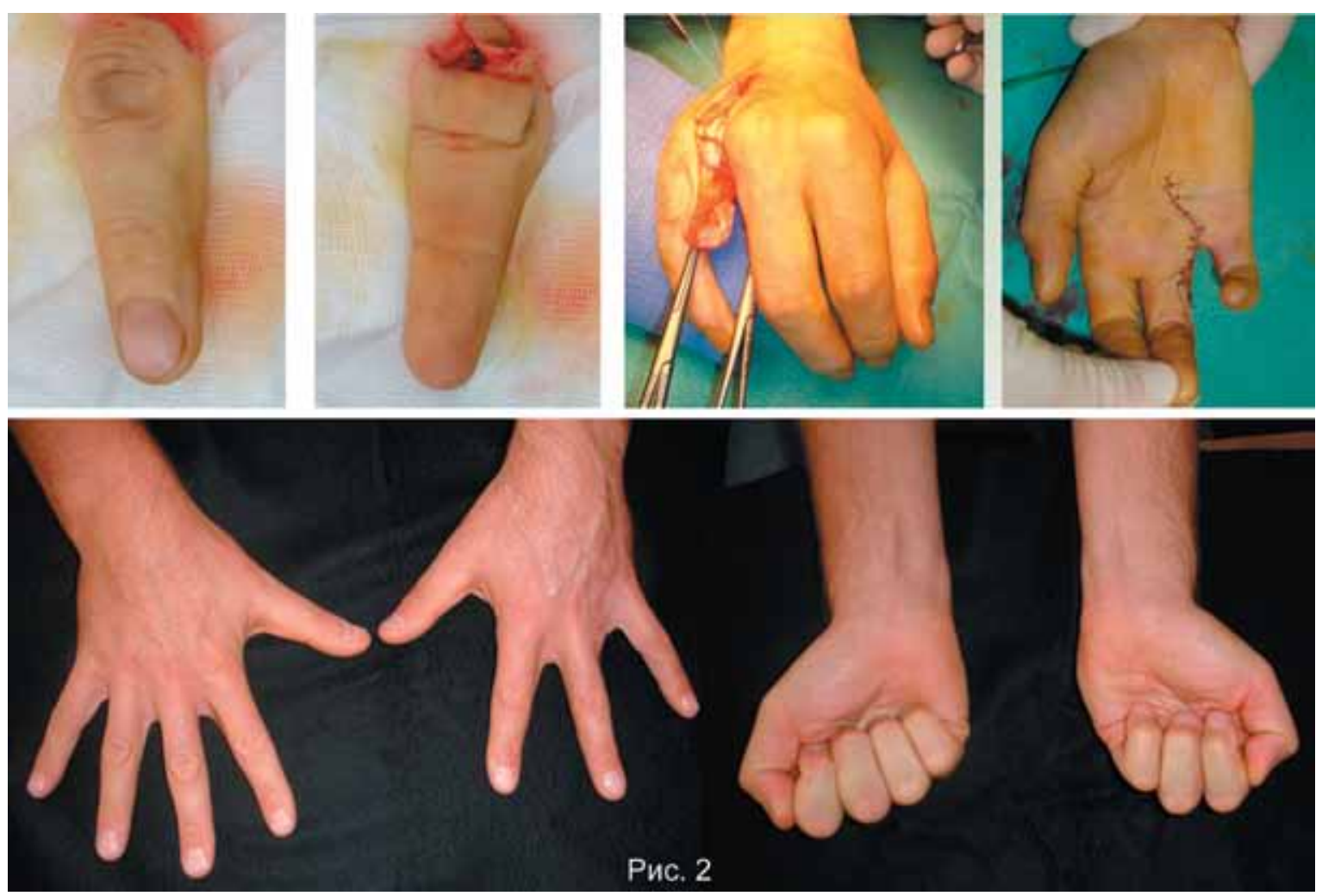
Такое вмешательство позволяет значительно сократить время реабилитации и количество осложнений, а также воссоздать гармоничную кисть. Если речь идет опациентах, у которых профессиональная деятельность связана с тяжелой ручной работой, то сохранение основной фаланги дает большее сохранение функции и силы [6], в таких случаях методика рекомендована; для закрытия раны часто требуется использование лоскутного трансплантата.

\section{2. Хирургическая техника}

Перед реплантацией проводится базовая подготовка фрагмента. Она хорошо описана Foucher [7], он рекомендует разрез 1,5 см по боковой поверхности пальца относительно межфалангового дистального сустава для подготовки фрагмента с целью сохранения его микрососудистого кровоснабжения (рис. 3).
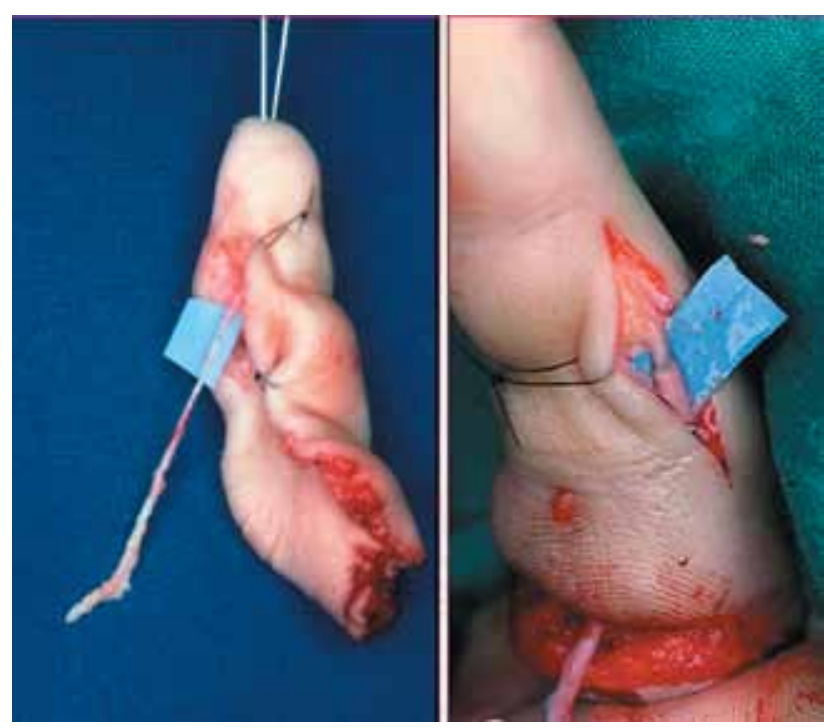

Рис. 3

На практике мы часто продлеваем разрез на всем протяжении окружающих мягких тканей. При этом гораздо легче выполняется подготовка кости, нервов, сосудов. Однако следует скрупулезно придерживаться рекомендаций Foucher по выполнению рассечения одной из двух артерий, чтобы избежать некроза мягкотканных покровов проксимального отдела пальца (рис. 4).

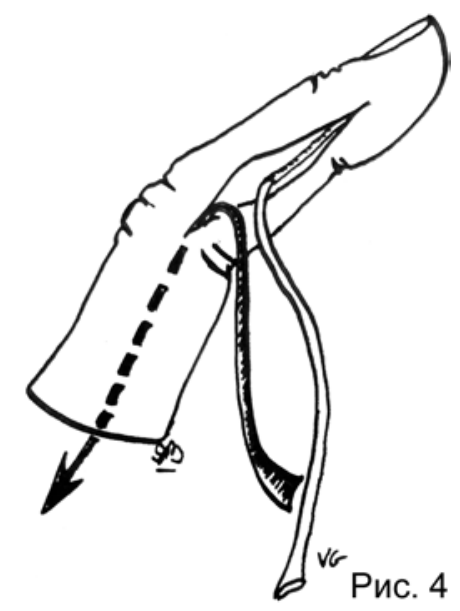

\section{1 Костно-сухожильный этап}

- В случае ампутации на уровне дистального межфалангового сустава артродез обязателен.

- При чресшеечной ампутации средней фаланги
Однако у детей попытка выполнить реплантацию возможна при стадии 4а. Реплантация открывает большие возможности, а через некоторое время, чтобы восстановить движения пальца, можно сделать пересадку сухожилия. Для этого во время реплантации необходимо располагать стержнем Hunter и поддерживать пассивно хорошую функцию сустава, чтобы подготовить последующую трансплантацию сухожилия.

достаточно на период достижения консолидации выполнить тенодез глубокого сгибателя в сочетании с временным артроризом дистального межфалангового сустава путем проведения дублированных спиц.

В этих двух случаях поверхностный сгибатель выступает единственным анатомическим образованием, обеспечивающим сгибание (рис. 5).

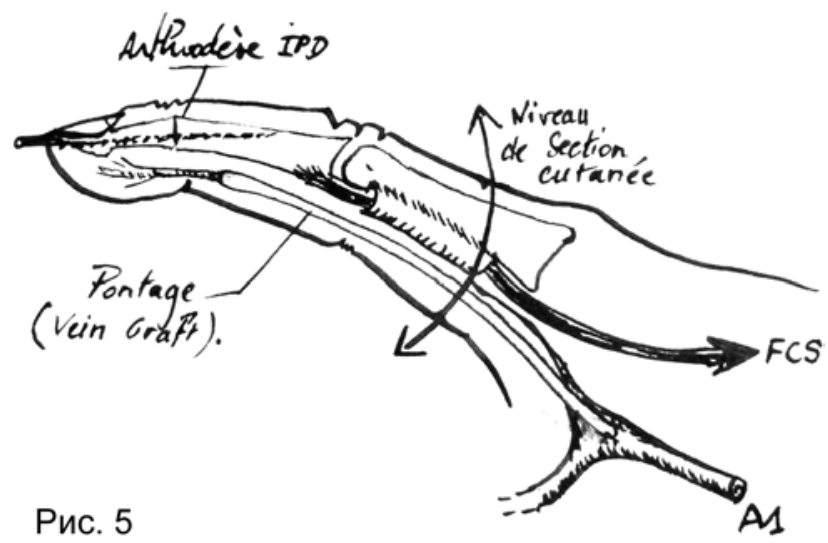

Следует немедленно выполнить мобилизацию проксимального межфалангового сустава во избежание развития его ригидности и восстановить кровоснабжение при подготовке артерий на скальпированном пальце.

\section{2 Подготовка сосудов}

\subsection{1 Этап подготовки артерий}

При скальпировании пальца кисти редко встречается разрыв двух артерий на одном уровне. Предпочтительнее использовать более короткую культю артерии (рис. 6).

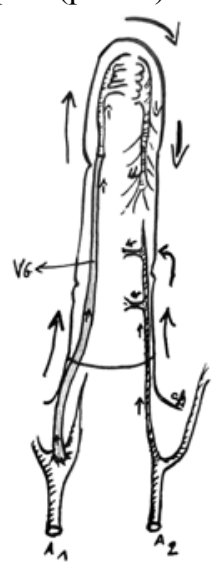

$A_{1}$ : vasuelarisation
da Fourreau
cutané via le
poutage.

$A_{2}$ : vascularisation de e'Iip et du FCS plus longue du squelefle deshabillé. (xe poste dissequer)

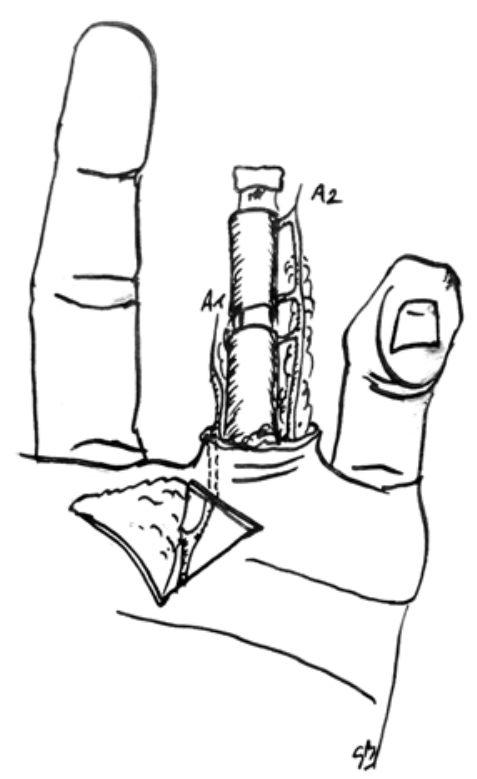

Рис. 6 
Ее выделяют на уровне бифуркации с другой коллатеральной артерией для выполнения шунтирования проксимального анастомоза. Если более длинная артерия не повреждена, то для сохранения васкуляризации межфалангового сустава и поверхностного разгибателя ее шунтируют на уровне бифуркации.

В окружающем мягкотканном футляре необходимо выбрать более длинную артерию и более короткую, расположенную на культе кисти. Используя эту артерию для дистального анастомоза, мы почти уверены, что не получим повреждений интимы на уровне дистального межфалангового сустава. Кровь, пройдя через ладонную дугу, осуществит питание мягкотканного компонента в отличие от коллатеральной артерии, которую не следует рассекать никогда (рис. 7).
При выборе венозного трансплантата для шунтирования необходимо учитывать его длину, размер и незначительное количество разветвлений. Обычно используется поверхностная вена предплечья. Однако требуемая длина часто превышает 8 см. Больший диаметр вены создает определенное несоответствие, поскольку трансплантат должен быть развернут с целью сохранения направления клапанов. Таким образом, выполняется анастомоз вены большего диаметра с артерией меньшего диаметра (на уровне второй фаланги). Чтобы избежать указанного нежелательного момента Grimaud et al. [8] предлагают использовать сопутствующие лучевой артерии вены, имеющие достаточно большие размеры и малую извилистость. Особенностью этих вен является их постоянный размер (рис. 8).
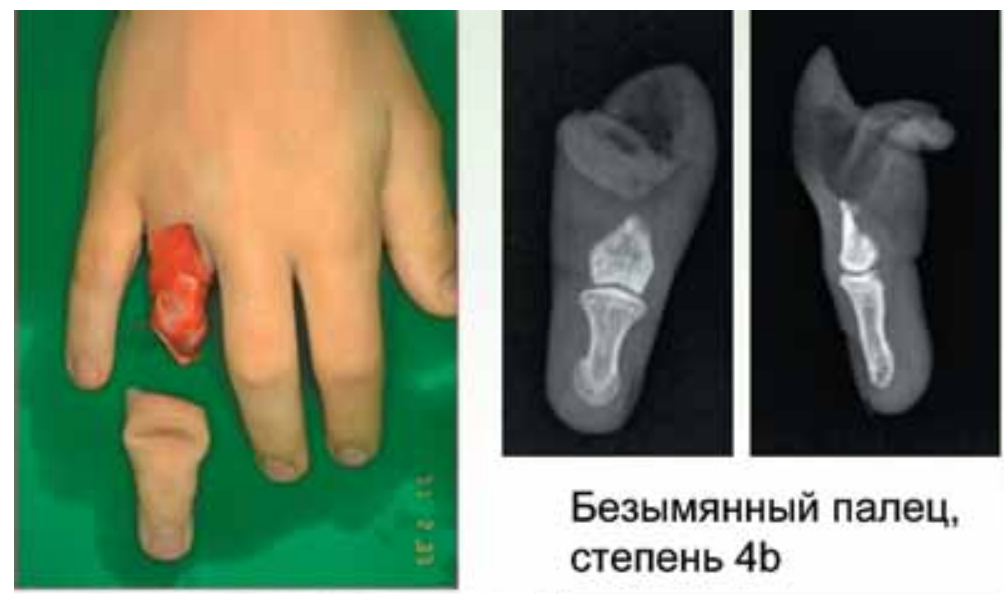

\section{Безымянный палец, степень 4b}
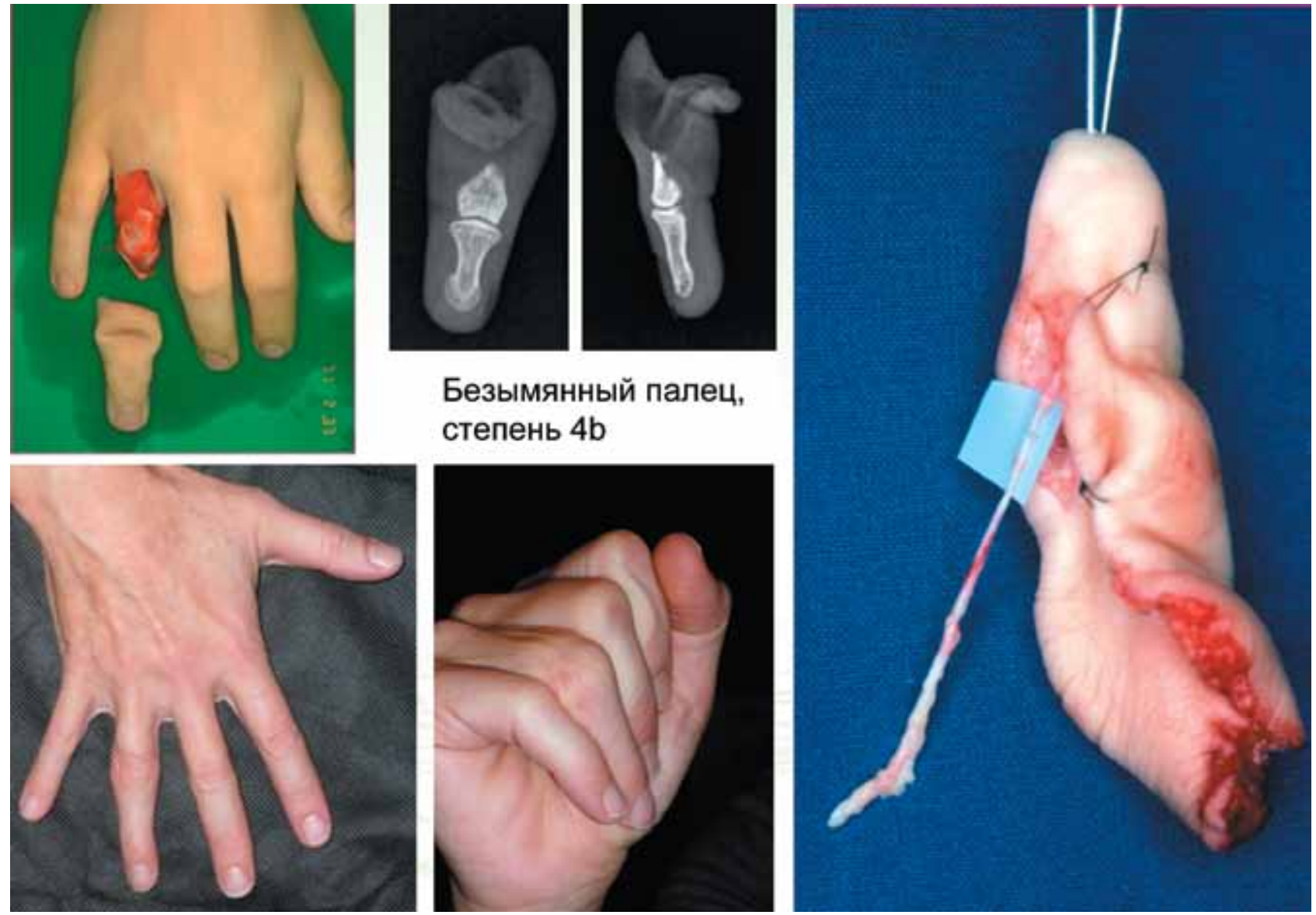

Рис. 7.

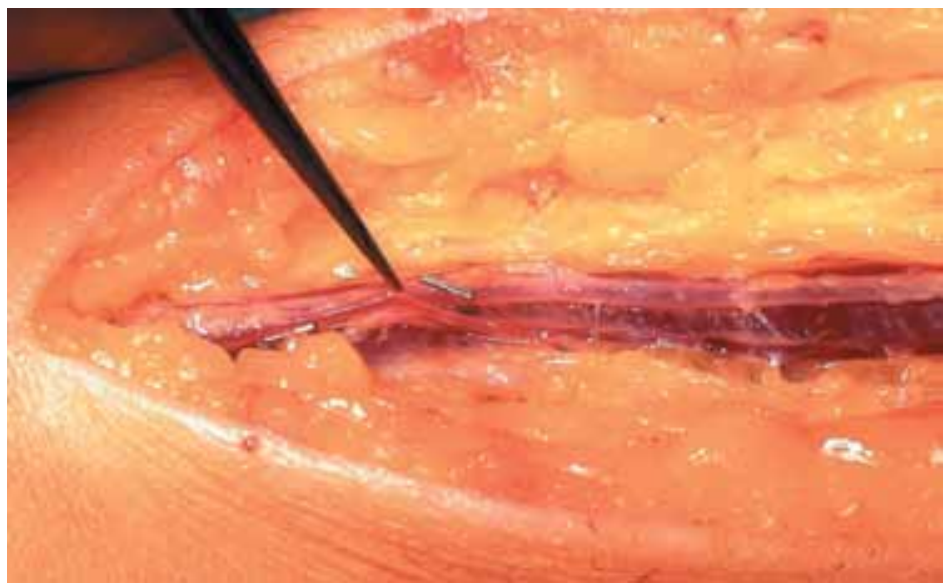

Рис. 8. 


\subsection{2 Этапь подготовки вен}

Одна или две вены рассекаются, разворачиваются, затем выполняется их непосредственный анастомоз (в отличие от артерий), это делается из-за укорочения кости и более значимого повреждения сосудов на тыльной стороне. Когда это невозможно, следует использовать васкуляризированный лоскут в форме “флага” (рис. 9, 10).

\section{3 Этапы подготовки нервов}

Непосредственная подготовка нервов иногда является невозможной. Использование одного трансплантата за счет латеральной кожной ветви предплечья в случае наличия дефекта является хорошим решением, но результат в плане восстановления чувствительности остается неутешительным [9-11]. Протяженность и вариабельность аксональных повреждений остаются неизвестными, несмотря на то, что на макроскопическом уровне концы нерва кажутся мало поврежденными. Прямое перекрестное сшивание - это хороший компромисс, если ситуация позволяет, его следует выполнять. К тому же, нужно подчеркнуть, что сопутствующие боли или болезненная неврома появляются очень редко [7] .

\section{4 Закрытие раны}

Часто рана закрывается непосредственно имеющимися тканями. Однако, если кожный чехол был рассечен по всей длине, что не позволяет закрыть рану, предпочтение отдается трансплантации кожи с боковой поверхности пальца или простому управляемому рубцеванию (при отсутствии экспозиции шунтирования), что позволяет профилактировать ишимизацию тканей вследствие отека. Напомним, что использование васкуляризированного кожного лоскута в виде "флага" при пластике дефектов кожи пальцев кисти (рис. 9, 10) очень полезно при решении проблем покрытия и венозного оттока от дорсальной поверхности пальца.

Этот прием обычно используется, если не выполнялось укорочения кости (резекция на уровне шейки второй фаланги или скальпирование без повреждения кости).


Рис. 9.

Stephane Barbary Hier à 19:33

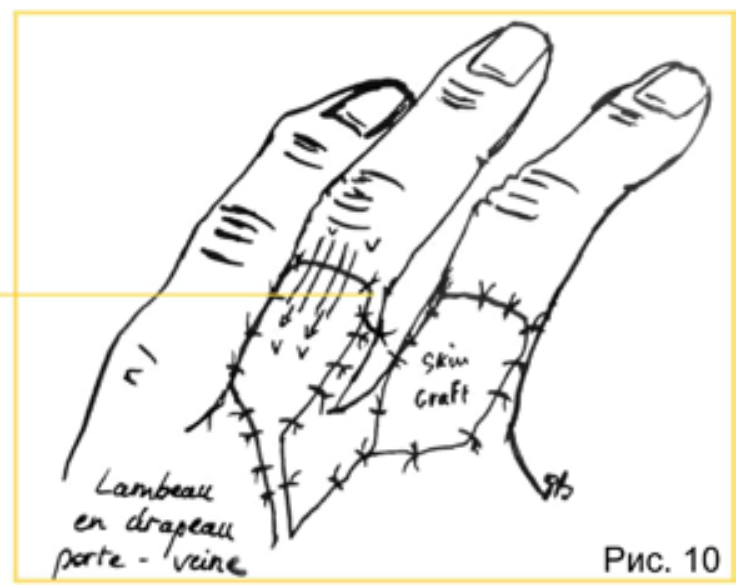




\section{3. Результаты по восстановлению кровоснабжения, функции и чувствительности}

\section{1 Степень выживаемости пальцев}

Степень выживаемости реплантированного пальца, главным образом, зависит от механизма ампутации. Отрыв и сдавление дают самые плохие результаты [12]. Изучение серий больных показывает, что шансы на выживание реплантированного пальца после полного отрыва составляют от 25 до $100 \%$ [3, 4, 7, 13-20], но данные последнего мета-анализа [12] свидетельствуют о 66 \% с возрастанием доли выживаемости при тракционном отчленении трехфалангового пальца (78 \%) в сравнении с первым (68\%). Доля выживаемости пальцев при неполном тракционном отчленении, по данным этого же исследования, составляет $78 \%$, в том числе большого пальца - 53 \% и 87 \% - длинных пальцев. Мы думаем, что в педиатрии эти цифры требуют отдельного уточнения.

Yan Lefevre et al. [20] на серии, включающей 23 случая тракционного отчленения пальцев у детей, средний возраст которых составлял 11 лет (от 2 до 15 лет), получил всего 25 \% успешных результатов по восстановлению кровоснабжения. Согласно исследований Urbaniaka, в группах 2 и 3 выживаемость составила $75 \%$ и $5 \%$ соответственно. Исследования подтверждают, что результаты у детей хуже, чем у взрослых. Это связывают с большей сложностью технического исполнения, обусловленной мелкими размерами сосудов, а также чаще встречающимися у детей спазмами сосудов. Подобные факты уже сообщались и другими авторами [22].

\section{2 Функциональные результаты}

Мы рассматриваем только случаи тракционного отчленения пальцев, сопровождающиеся повреждениями костей и суставов. Полный диапазон измерений
TAM (Total Arc of Motion), относящийся к суммированной амплитуде движения всех суставов пальца, по данным исследований, составляет от $70^{\circ}$ до $200^{\circ}$. По результатам мета-анализа по 10 сериям из 75 пациентов, проведенного Sears et al. [21], среднее значение ТАМ составляет 174 градуса. Это значение очень близко к известным данным по самым большим сериям исследования $[4,14,18]$, к тому же, оно кажется особенно достойным доверия. Надо отметить, что дистальный межфаланговый сустав часто блокирован (вследствие артродеза или тенодеза).

Следовательно, сохранение и поддержание подвижности в проксимальных межфаланговых суставах является основополагающим принципом. Чтобы избежать деградации в отдаленном периоде вследствие остеохондронекроза, следует как можно раньше начинать разработку проксимальных межфаланговых суставов и сохранять их кровоснабжение в момент реимплантации [23].

\section{3 Результатьи по восстановлению чувстви-} тельности

Тракционный механизм повреждения дает гораздо худшие результаты по восстановлению чувствительности после реимплантации, чем после прямой ампутации [24]. Естественно, что аксональные повреждения являются более значительными, чего не видно при осмотре, даже под микроскопом. Тест Weber (2PD), по данным разных авторов [7, 14, 18, 25], варьирует от 9 мм до 14 мм, где среднее значение составляет 13 мм, согласно мета-анализу Sears et al. [21]. Молодой возраст пациентов в момент травмы благотворно сказывается на результатах лечения по восстановлению чувствительности $[18,24]$.

\section{ЗАКЛЮЧЕНИЕ}

Травмы пальцев в результате тракционного отчленения кольцом представляют трудности для хирургического лечения. Повреждения питающих сосудов являются более обширными, чем кажется клинически, а риск ошибки очень высок, если иссечение поврежденной части сосудов выполнено недостаточно. Восстановление только одного из двух артериальных лучей необходимо выполнять обязательно в случае полного тракционного отчленения пальца или ишемии без по- вреждения скелета. Для улучшения результата лечения всегда необходимо сохранять кровоснабжение в проксимальном межфаланговом суставе скальпированного пальца. Несмотря на эти рекомендации, прогноз сосудистого и функционального восстановления остается менее отчетливым, по сравнению со случаями прямого отсечения $[2,7,26]$, где среднее значение выживаемости пальца составляет $66 \%$ в случае полного тракционного отчленения и $78 \%$ - в случае неполного.

\section{ЛИТЕРАТУРA (REFERENCES)}

1. Urbaniak J.R., Evans J.P., Bright D.S. Microvascular management of ring avulsion injuries // J. Hand Surg. Am. 1981. Vol. 6, No 1. P. 25-30.

2. Review of 30 digital ring avulsions / M. Schoofs, P. Leps, F. Millot, H. Migaud // Ann. Chir. Main Memb. Super. 1990. Vol. 9, No 4. P. 245-251.

3. Kay S., Werntz J., Wolff T.W. Ring avulsion injuries: classification and prognosis // J. Hand Surg. Am. 1989. Vol. 14, No 2. Pt 1. P. 204-213.

4. Ring avulsion injuries: microsurgical management / R. Adani, C. Castagnetti, R. Busa, A. Caroli // J. Reconstr. Microsurg. 1996. Vol. 12, No 3. P. 189-194.

5. 5.Primary ray resection as an alternative to microsurgical replantation in the management of ring finger avulsion / J. Segret, S. Barbary, D. Pétry, G. Dautel // Chir. Main. 2008. Vol. 27, No 5. P. 202-207.

6. Results of ray resection and amputation for ring avulsion injuries at the proximal interphalangeal joint / E. Nuzumlali, E. Orhun, K. Oztürk, S. Cepel, S. Polatkan // J. Hand Surg. Br. 2003. Vol. 28, No 6. P. 578-581.

7. Revascularization of digital avulsion injuries caused by rings / G. Foucher, N. Citron, M. Merle, M. Dury // Ann. Chir. Main. 1986. Vol. 5, No 3. P. 256-259.

8. Grimaud O., Delpit X., Hardy P. An optimum donor site for venous grafting for microsurgery // Chir. Main. 2011. Vol. 30, No 3. P. 205-210.

9. Recovery of sensation in replanted digits - time of recovery and degree of two-point discrimination / S. Yamauchi, S. Nomura, M. Yoshimura, T. Ueno, Y. Iwai, K. Shimamura // J. Microsurg. 1982. Vol. 3, No 4. P. 206-213.

10. Molski M. Replantation of fingers and hands after avulsion and crush injuries // J. Plast. Reconstr. Aesthet. Surg. 2007. Vol. 60, No 7. P. 748-754.

11. Sebastin S.J., Chung K.C. A systematic review of the outcomes of replantation of distal digital amputation // Plast. Reconstr. Surg. 2011. Vol. 128, No 3. P. 723-737.

12. Results of 1018 digital replantations in 552 patients / S. Waikakul, S. Sakkarnkosol, V. Vanadurongwan, A. Un-nanuntana // Injury. 2000. Vol. 31, No 1. P. 33-40.

13. Thumb avulsion: results of replantation/revascularization / E.J. Bieber, M.B. Wood, W.P. Cooney, P.C. Amadio // J. Hand Surg. Am. 1987. Vol. 12, No 5. Pt. 1. P. 786-790.

14. Review of 30 digital ring avulsions / M. Schoofs, P. Leps, F. Millot, H. Migaud // Ann. Chir. Main Memb. Super. 1990. Vol. 9, No 4. P. $245-251$.

15. Avulsion injuries of the thumb / M.V. Stevanovic, C. Vucetic, M. Bumbasirevic, C. Vuckovic // Plast. Reconstr. Surg. 1991. Vol. 87, No 6. P. 1099-1104. 
16. Lim B.H., Tan B.K., Peng Y.P. Digital replantations including fingertip and ring avulsion // Hand Clin. 2001. Vol. 17, No 3. P. 419-431, viii-ix.

17. Akyürek M., Safak T., Keçik A. Ring avulsion replantation by extended debridement of the avulsed digital artery and interposition with long venous grafts // Ann. Plast. Surg. 2002. Vol. 48, No 6. P. 574-581.

18. Analysis of prognostic factors in ring avulsion injuries / M. Sanmartín, F. Fernandes, A.S. Lajoie, A. Gupta // J. Hand Surg. Am. 2004. Vol. 29 , No 6. P. 1028-1037.

19. Ring avulsion: injury pattern, treatment, and outcome / D. Brooks, R.F. Buntic, G.M. Kind, K. Schott, G.M. Buncke, H.J. Buncke // Clin. Plast. Surg. 2007. Vol. 34, No 2. P. 187-195,viii.

20. Digital avulsion with compromised vascularization: study of 23 cases in children / Y. Lefèvre, C. Mallet, B. Ilharreborde, P. Jehanno, J.M. Frajmann, G.F. Penneçot, K. Mazda, F. Fitoussi // J. Pediatr. Orthop. 2011. Vol. 31, No 3. P. 259-265.

21. Sears E.D., Chung K.C. Replantation of finger avulsion injuries: a systematic review of survival and functional outcomes // J. Hand Surg. Am. 2011. Vol. 36, No 4. P. 686-694.

22. Digital replantation in children: a long-term follow-up study / K. Ikeda, S. Yamauchi, F. Hashimoto, K. Tomita, M. Yoshimura // Microsurgery. 1990. Vol. 11, No 4. P. 261-264.

23. Aseptic bone necrosis following reimplantation of a degloving finger / L. Obert, H.P. Corral, F. Gérard, A. Jarry, P. Garbuio, Y. Tropet // Chir. Main. 2002. Vol. 21, No 1. P. 36-40.

24. Glickman L.T., Mackinnon S.E. Sensory recovery following digital replantation // Microsurgery. 1990. Vol. 11, No 3. P. $236-242$.

25. Long-term results of replantation for complete ring avulsion amputations / R. Adani, I. Marcoccio, C. Castagnetti, L. Tarallo // Ann. Plast. Surg. 2003. Vol. 51, No 6. P. 564-568; discussion 569.

26. Replantation of ring avulsion of index, long, and ring fingers / O.F. Tseng, Y.C. Tsai, F.C. Wei, D.A. Staffenberg // Ann. Plast. Surg. 1996. Vol. 36 , No 6. P. 625-628.

Рукопись поступила 22.11.2013

Сведения об́ авторе:

Barbary Stephane - Университет Анри Поанкаре (Henry Poincaré), профессор по специальностям "Микрохирургия", "Хирургия кисти", MD, Phd, , Nancy, France.
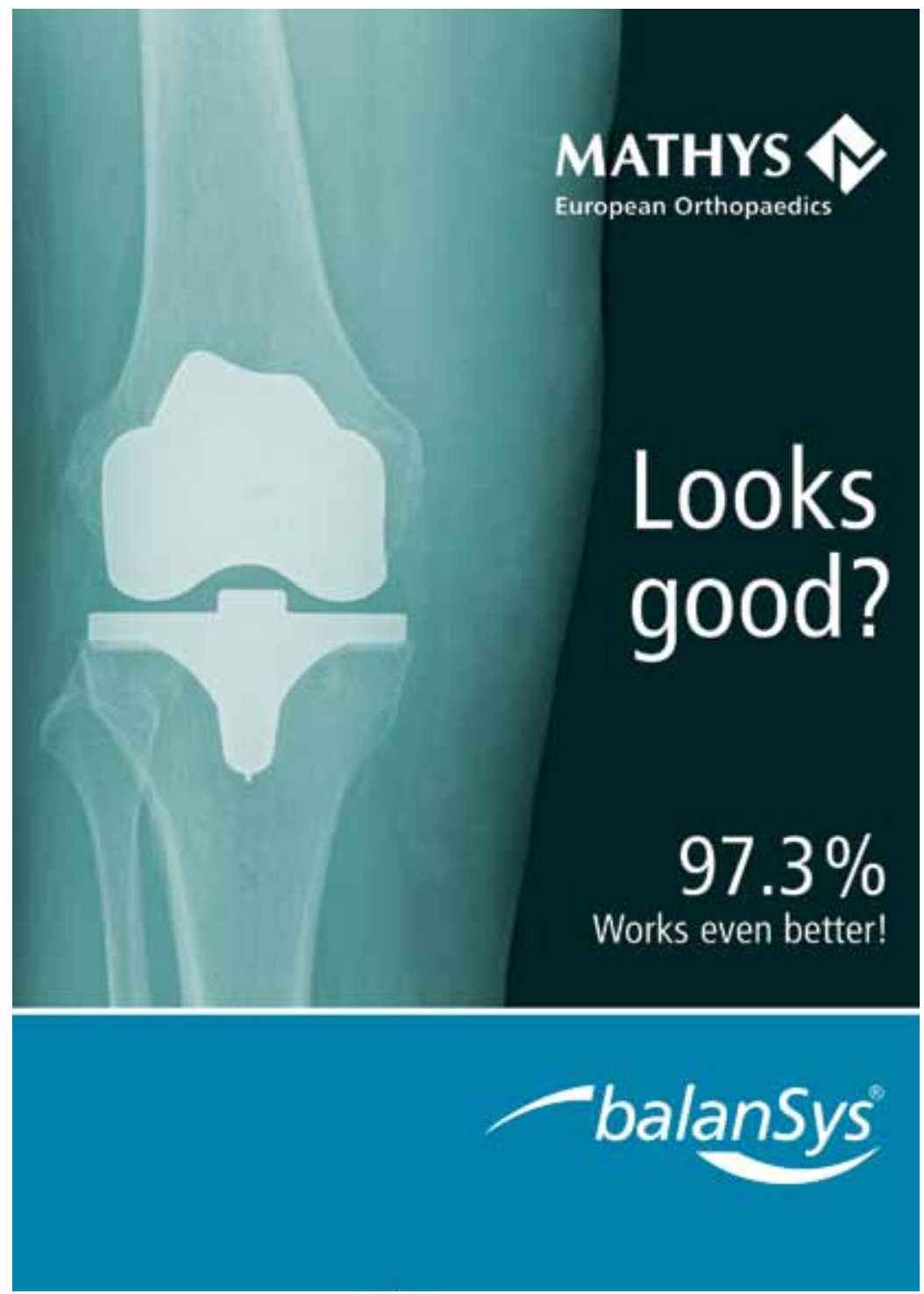